\section{Senescence impairs successful reprogramming to pluripotent stem cells}

\author{
Ana Banito, ${ }^{1}$ Sheikh T. Rashid, ${ }^{2}$ Juan Carlos Acosta, ${ }^{1}$ \\ SiDe Li, ${ }^{3,4}$ Carlos F. Pereira, ${ }^{5}$ Imbisaat Geti, ${ }^{2}$ \\ Sandra Pinho, ${ }^{6}$ Jose C. Silva, ${ }^{7}$ Veronique Azuara, ${ }^{6}$ \\ Martin Walsh, ${ }^{3,4}$ Ludovic Vallier, $^{2}$ and Jesús Gil ${ }^{1,8}$ \\ ${ }^{1}$ Cell Proliferation Group, MRC Clinical Sciences Centre, \\ Faculty of Medicine, Imperial College, London W12 0NN, \\ United Kingdom; ${ }^{2}$ Laboratory for Regenerative Medicine, \\ University of Cambridge, Department of Surgery, Cambridge \\ CB2 0SZ, United Kingdom; ${ }^{3}$ Department of Pediatrics, Mount \\ Sinai School of Medicine, New York, New York 10029, USA; \\ ${ }^{4}$ Department of Structural and Chemical Biology, Mount Sinai \\ School of Medicine, New York, New York 10029, USA; \\ ${ }^{5}$ Lymphocyte Development Group, MRC Clinical Sciences \\ Centre, Faculty of Medicine, Imperial College, London W12 \\ ONN, United Kingdom; ${ }^{6}$ Institute of Reproductive and \\ Developmental Biology, Faculty of Medicine, Imperial College, \\ London W12 0NN, United Kingdom; ${ }^{7}$ Wellcome Trust Centre \\ for Stem Cell Research, University of Cambridge, Cambridge \\ CB2 1QR, United Kingdom
}

Somatic cells can be reprogrammed into induced pluripotent stem (iPS) cells by overexpressing combinations of factors such as Oct4, Sox2, Klf4, and c-Myc. Reprogramming is slow and stochastic, suggesting the existence of barriers limiting its efficiency. Here we identify senescence as one such barrier. Expression of the four reprogramming factors triggers senescence by up-regulating p53, p16 ${ }^{\mathrm{INK} 4 \mathrm{a}}$, and $\mathrm{p} 21^{\mathrm{CPP} 1}$. Induction of DNA damage response and chromatin remodeling of the INK4a/ARF locus are two of the mechanisms behind senescence induction. Crucially, ablation of different senescence effectors improves the efficiency of reprogramming, suggesting novel strategies for maximizing the generation of iPS cells.

Supplemental material is available at http://www.genesdev.org.

Received April 14, 2009; revised version accepted July 31, 2009.

The ability to derive pluripotent cells from somatic cells may fulfill the promise of unlimited stem cells for applications in basic biology, drug development, or regenerative medicine. Different strategies have been used to reprogram somatic cells into pluripotent stem cells. These include nuclear transfer (Wilmut et al. 1997), cellular fusion with embryonic stem (ES) cells (Ying et al. 2002), or the expression of factors linked to stem cell pluripotency (Takahashi and Yamanaka 2006). Re-

[Keywords: iPS; senescence; reprogramming; miR-302; Sox2; p21 ${ }^{\mathrm{CIP} 1}$ ] ${ }^{8}$ Corresponding author.

E-MAIL jesus.gil@csc.mrc.ac.uk; FAX 44-20-8383-8306.

Article published online ahead of print. Article and publication date are online at http://www.genesdev.org/cgi/doi/10.1101/gad.1811609. garding the latter, Takahasi and Yamanaka (2006) first showed that mouse somatic cells could be reprogrammed to a pluripotent-like state by expressing four transcription factors (Oct4, Sox2, Klf4, and c-Myc). Since then, induced pluripotent stem (iPS) cells have been derived from cells of multiple origin, including human adult fibroblast and keratinocytes (Takahashi et al. 2007; Yu et al. 2007; Lowry et al. 2008; Park et al. 2008). Alternating the combination of factors (i.e., substituting Klf4 and c-Myc by Nanog and Lin28) has been used successfully to reprogram different cells or improve the efficiency of reprogramming (Yu et al. 2007; Feng et al. 2009). Currently, the two major limitations for translating iPS-based reprogramming into clinical application are to routinely deliver iPS cells safe and free of vector and transgene sequences, and to improve the reprogramming efficiency. The first issue is being addressed by using alternative methods to deliver the reprogramming factors such as transient transfection (Okita et al. 2008), transposons (Kaji et al. 2009), adenoviral (Stadtfeld et al. 2008), episomal vectors (Yu et al. 2009), or recombinant proteins (Zhou et al. 2009). Different strategies are under investigation to enhance the efficiency of reprogramming (Feng et al. 2009).

Reprogramming occurs as a gradual process; for example, upon retroviral transduction, lineage-specific genes are silenced gradually and, as embryonic markers become activated, endogenous genes linked to pluripotency are induced (Brambrink et al. 2008). However, this process results in only a small percentage of the infected population of cells becoming pluripotent. This suggests that barriers could be limiting the efficiency of successful reprogramming. The comparison of genetic and epigenetic profiles of partially reprogrammed cells (Pre-iPS) and iPS show that independently derived Pre-iPS share similar gene expression and epigenetic profiles, reflecting a common intermediate state (Mikkelsen et al. 2008; Sridharan et al. 2009). Pre-IPS display incomplete epigenetic remodeling and persistent DNA hypermethylation, and can be converted into iPS with varied efficiency through global inhibition of DNA methylation (Mikkelsen et al. 2008). The existence of other mechanisms limiting reprogramming efficiency remains to be investigated.

Senescence is the irreversible arrest during the G1 transition of the cell cycle elicited by replicative exhaustion or in response to stresses such as DNA damage, chemotherapeutic drugs, or aberrant expression of oncogenes. This arrest is implemented primarily through activation of p53 and the up-regulation of the cyclindependent kinase (CDK) inhibitors $16^{\mathrm{INK} 4 \mathrm{a}}$ and $\mathrm{p} 21^{\mathrm{CIP} 1}$ (Collado et al. 2007). Beyond being simply an in vitro observation, senescence plays relevant physiological roles. In particular, oncogene-induced senescence (OIS) is widely observed among varied premalignant lesions and is believed to be tumor-suppressive. Senescence also limits the homeostasis of normal tissues through the regulation of stem cell self-renewal, therefore impacting on aging (Collado et al. 2007).

In this report, we investigate the direct relationship between the process of iPS reprogramming and senescence. We observe that reprogramming initially triggers a stress response with characteristics of senescence. This reprogramming-induced senescence (RIS) acts as an 
initial barrier limiting the efficiency of the process. Decisively, alleviating senescence in these cultures enhances reprogramming efficiency.

\section{Results and Discussion}

We analyzed the early response of somatic cells to expression of the four reprogramming factors (Oct4, Sox2, Klf4, and c-Myc) (Fig. 1A). To this end, a polycistronic cassette encoding Oct4, Sox2, Klf4, and c-Myc (OSKM) (Carey et al. 2009) was transferred to a retroviral vector (pBABE-OSKM). Early events after the infection of IMR90 human fibroblasts with the OSKM vector (Fig. 1) or coinfection with four individual vectors expressing the reprogramming factors (4-F) (Supplemental Fig. S1) were monitored. As controls, we included cells infected with an activated version of RAS (H-RAS ${ }^{\mathrm{G} 12 \mathrm{~V}}$ ), able to cause senescence. Upon expression of the reprogramming factors, we observed a decrease in the numbers and growth of IMR90 cells as evaluated by growth curves, colony formation assays, and a decrease in the percentage of cells incorporating BrdU (Fig. 1B-D). Expression of the reprogramming factors increased the percentage of cells arrested in G1, without inducing apoptosis significantly in our experimental conditions (Supplemental Fig. 1). Cells expressing the four reprogramming factors resembled senescent cells with an enlarged cytoplasm and displayed senescent-associated $\beta$-galactosidase (SA $\beta$-Gal) activity, and senescence-associated heterochromatic foci (SAHF) (Fig. 1E; Supplemental Fig. 1). Expression of reprogramming factors in another strain of human fibroblasts (BJ) or in mouse embryonic fibroblasts (MEFs) also resulted in a growth arrest with characteristics of senescence (Supplemental Fig. 2). To dissect how each of the reprogramming factors contributed to senescence, we infected IMR90 cells with each factor separately. To our surprise, each of the four factors-Oct4, Sox2, Klf4, and cMyc-diminished cell growth, decreased BrdU incorporation, and up-regulated markers and characteristics of senescence in IMR90 cells (Supplemental Fig. S3), thus suggesting a complex scenario in which different pathways could be activated simultaneously to trigger senescence in response to their combined expression.

To identify the pathways responsible for senescence induction at the early stages of reprogramming, IMR90 cells were infected with the OSKM vector and analyzed by immunofluorescence. Expression of reprogramming factors caused a DNA damage response as observed by elevated levels of nuclei staining positive with the pST/Q or $\gamma \mathrm{H} 2 \mathrm{AX}$ antibodies (Fig. 2A; data not shown). Interestingly, OSKM expression also caused oxidative stress, as reflected by elevated levels of the oxidized base 8oxoG (Supplemental Fig. S5A). We also observed a significant upregulation of the tumor suppressors $\mathrm{p} 16^{\text {INK4a }}$ p53, and $\mathrm{p} 21^{\mathrm{CIP} 1}$-critical senescent effectors-upon infection with the OSKM polycistronic vector in IMR90 cells (Fig. 2B-D; Supplemental Fig. 5), BJ, and MEFs (Supplemental Fig. S2). Reanalysis of published transcriptional profiles of MEFs expressing the four factors under inducible control (Mikkelsen et al. 2008) showed increased p16 ${ }^{\text {Ink4a }}$ and p2 $1^{\text {Cip1 }}$ levels that decline slightly at later times (Supplemental Fig. S6). A time course in IMR90 cells expressing the reprogramming factors showed that although levels of senescence effectors are elevated (compare with vector), at later times their expression decreases, especially for p53 and p $21^{\mathrm{CIP} 1}$ (Supplemental Fig. S7).

Interestingly, we also observed the up-regulation of p16 ${ }^{\mathrm{INK} 4 \mathrm{a}}$ and $\mathrm{p} 21^{\mathrm{CIP} 1}$ (Supplemental Fig. S8C) during heterokaryon-based reprogramming (Pereira et al. 2008), suggesting an inherent link between senescence and reprogramming. We reasoned that if up-regulation of senescence effectors was an early response to reprogramming, their levels could remain elevated in Pre-iPS cells. Indeed, expression of $\mathrm{p} 16^{\mathrm{INK} 4 \mathrm{a}}$ and $\mathrm{p} 21^{\mathrm{CIP} 1}$ was upregulated in Pre-iPS derived from MEFs when compared with controls (Supplemental Fig. S9A), and similar conclusions were drawn from re-examination of published data (Supplemental Fig. 9B,C; Mikkelsen et al. 2008; Sridharan et al. 2009). Therefore, induction of senescence upon reprogramming (RIS) is observed in different cell types, using different reprogramming approaches, and it is reflected by elevated levels of senescence effectors such as $\mathrm{p} 21^{\mathrm{CIP} 1}$ and $\mathrm{p} 16^{\mathrm{INK} 4 \mathrm{a}}$ in Pre-iPS.

To analyze the contribution of the senescence effectors up-regulated during RIS, we coexpressed the OSKM vector with the E6 and E7 proteins of HPV16, which inactivate the p53 and the $\mathrm{Rb}$ networks, respectively. As shown in Figure 2E, expression of either alleviates the growth arrest induced by the reprogramming factors, and joint expression of both E6 and E7 at least had additive effects. To test specifically for the contribution of
Oct4, Sox2,
Klf4, c-Myc

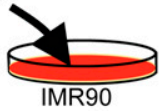

B

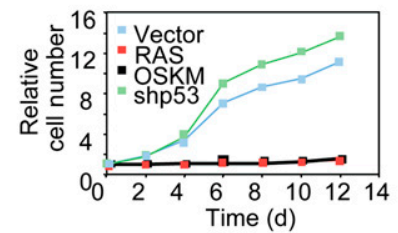

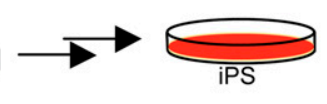

C

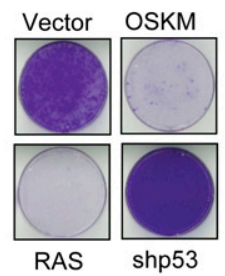

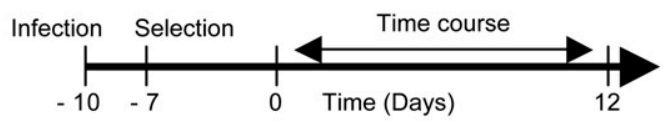

E

D

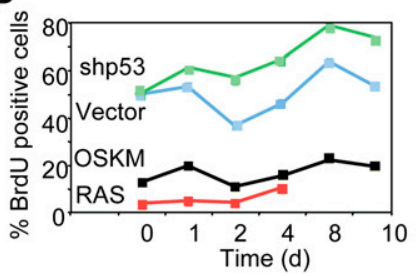

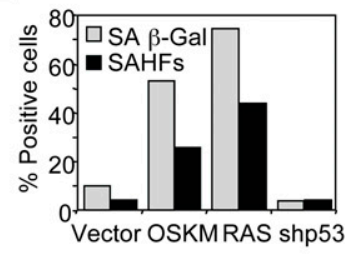

Figure 1. Induction of senescence upon expression of the four reprogramming factors. $(A)$ Timeline of the experiments in this figure and Figure 2. IMR90 human fibroblasts were infected with a polycistronic vector expressing the four factors (Oct4, Sox2, Klf4, and c-Myc). Cells were selected for $7 \mathrm{~d}$. (B) Effect of the expression of the four factors on the growth of IMR90 cells. (C) Crystal violet-stained plates of IMR90 cells infected with the indicated vectors. $(D)$ Expression of the four reprogramming factors causes a decrease in the percentage of IMR90 cells incorporating BrdU. $(E)$ Effect of the vector expressing the four reprogramming factors over SA $\beta$-Gal activity and SAHF formation in IMR90 cells. 

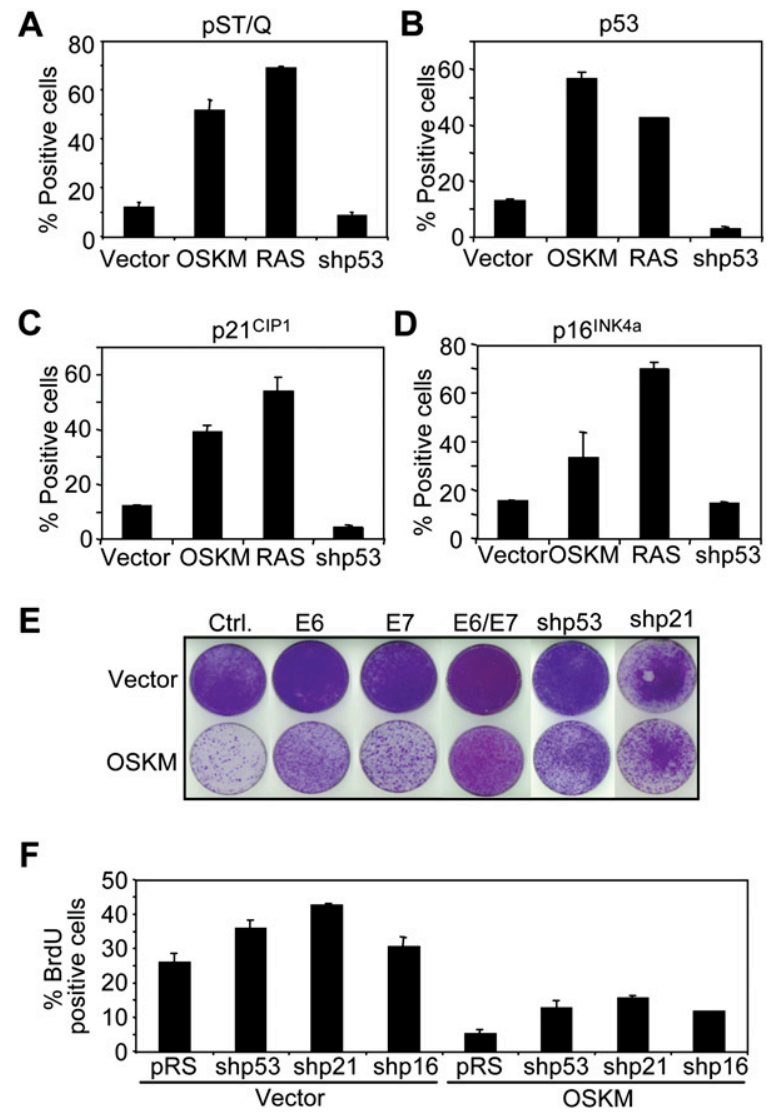

Figure 2. Molecular analysis of senescence induced by expression of reprogramming factors. $(A-D)$ Expression of the four reprogramming factors results in an increase in the percentage of cells positive for DNA damage (as measured by pST/Q IF), p53, p21 ${ }^{\mathrm{CIP1}}$, and $\mathrm{p} 16^{\mathrm{INK} 4 \mathrm{a}}$. An explanation of the procedures used to calculate the percentage of positive cells is detailed in the Supplemental Material and in Supplemental Figure 4. $(E, F)$ The ability of the four reprogramming factors to block proliferation of IMR90 cells is alleviated by coexpression of HPV E6 and/or HPV E7 or knockdown of p53, $\mathrm{p} 16^{\mathrm{INK} 4 \mathrm{a}}$, or $\mathrm{p} 21^{\mathrm{CIP} 1}$ using shRNAs as measured by crystal violet staining $(E)$ or BrdU incorporation $(F)$.

$\mathrm{p} 16^{\mathrm{INK} 4 \mathrm{a}}, \mathrm{p} 21^{\mathrm{CIP} 1}$, and $\mathrm{p} 53$ to the arrest, we used shRNAs. Analysis of the BrdU incorporation and growth in cells infected with the OSKM vector showed that $\mathrm{p} 53, \mathrm{p} 21^{\mathrm{CIP} 1}$, and $\mathrm{p} 16^{\mathrm{INK} 4 \mathrm{a}}$ are relevant for the arrest (Fig. 2E,F).

The INK4a/ARF locus is normally subjected to strong epigenetic repression mediated by H3K27 methylation and recruitment of Polycomb-repressive complexes. Therefore, we investigated the extent of H3K27 methylation at the INK4a/ARF locus in response to the expression of reprogramming factors. Chromatin immunoprecipitation (ChIP) was used to measure the ratio of trimethylated $\mathrm{H} 3 \mathrm{~K} 27$ (H3K27me3) to total $\mathrm{H} 3$ using primer sets described previously (Fig. 3A; Barradas et al. 2009). The repressive mark peaked around the INK4a promoter, and we observed a decrease in the levels of the $\mathrm{H} 3 \mathrm{~K} 27 \mathrm{me} 3$ modification around the INK4a/ARF locus in response to the expression of reprogramming factors (Fig. 3B). Human ES (hES) cells were included for comparison, showing even higher levels of H3K27me3. In parallel, we observed an increase in the $\mathrm{H} 3 \mathrm{~K} 4 \mathrm{me} 3$ marks around the INK4a/ARF locus upon expression of the reprogramming factors (Fig. 3C). To investigate the mechanism behind these chromatin changes, we analyzed the levels of the $\mathrm{H} 3 \mathrm{~K} 27 \mathrm{me} 3$ histone demethylase JMJD3. JMJD3 is a key contributor to the remodeling of the INK4a/ARF locus during senescence (Barradas et al. 2009). We observed an acute up-regulation of JMJD3 upon expression of the reprogramming factors (Fig. 3D,E). In agreement with this observation, ChIP reflected an increased recruitment of JMJD3 to the INK4a promoter; interestingly, we also observed a decrease in the binding of the H3K27me3 histone methylase EZH2 to the locus but only marginal effects on their total levels of expression (Fig. 3F; data not shown). We observed similar remodeling of the INK4a/ $A R F$ locus when the reprogramming factors were expressed from individual vectors (4-F) (Supplemental Fig. S10).

To understand how the $\mathrm{p} 53 / \mathrm{p} 21^{\mathrm{CIP} 1}$ pathway is engaged during RIS, we expressed each of the reprogramming factors individually. Sox2, Klf4, and c-Myc each upregulated $\mathrm{p} 21^{\mathrm{CIP} 1}$ levels (Fig. 4A). Interestingly, they induced $\mathrm{p} 21^{\mathrm{CIP} 1}$ by different mechanisms. Sox 2 expression resulted in a p53-independent up-regulation of p21 ${ }^{\mathrm{CIP1}}$, while c-Myc or Klf4 induced both p53 and p21 ${ }^{\mathrm{CIP} 1}$, although only $\mathrm{c}-\mathrm{Myc}$ provoked DNA damage
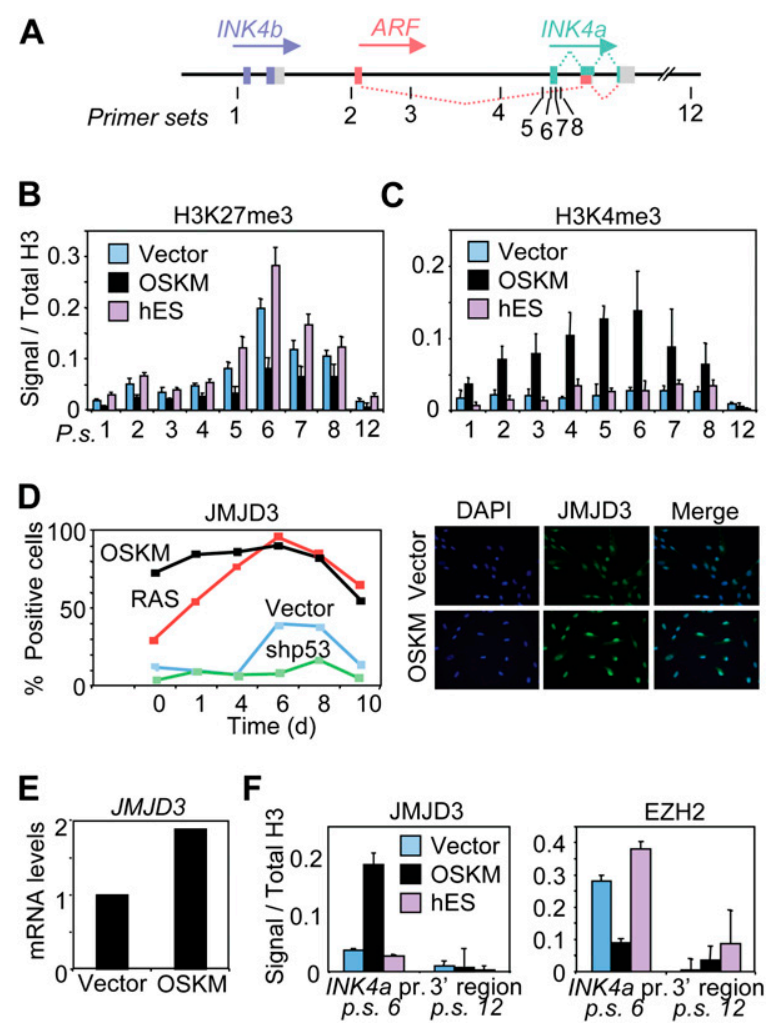

Figure 3. The histone demethylase JMJD3 contributes to the regulation of the INK4a/ARF locus during RIS. (A) Cartoon showing the organization of the human INK4b/ARF/INK4a locus and primer sets used. $(B, C)$ Expression of the four reprogramming factors results in a loss of H3K27me3 marks $(B)$ and an increase in H3K4me3 marks $(C)$ in the INK4b/ARF/INK4a locus. (D) The H3K27me3 histone demethylase JMJD3 is induced in response to the expression of the four reprogramming factors. (E) Up-regulation of JMJD3 transcript in response to the expression of the four reprogramming factors. $(F)$ ChIP analysis showing an enrichment of JMJD3 and a loss of EZH2 in the INK4a promoter in response to expression of the four reprogramming factors. 
A

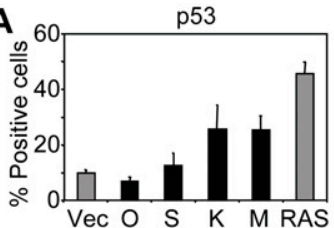

C

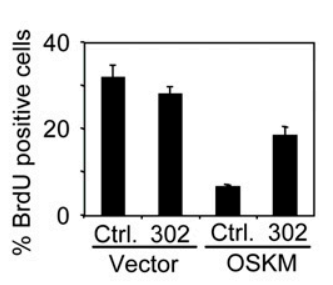

p21 CIP1a

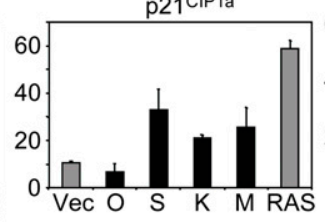

Ctrl. $\quad 302$

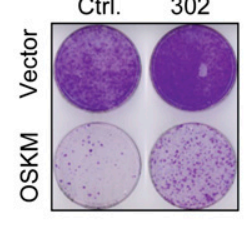

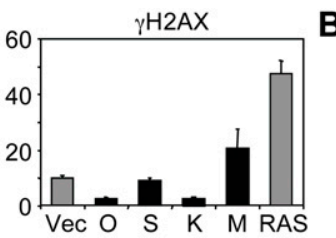

D

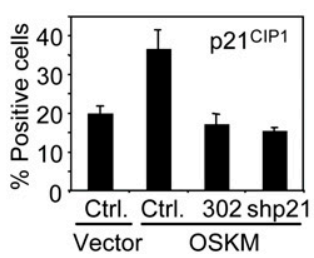

B

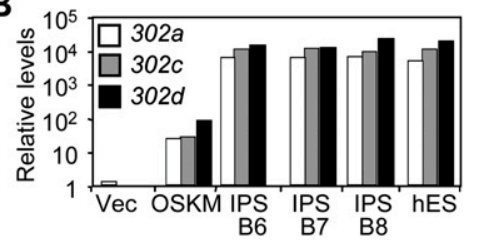

E

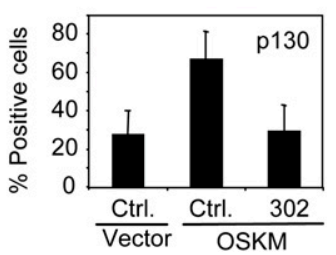

Figure 4. Role of $\mathrm{p} 21^{\mathrm{CIP} 1}$ during RIS. $(A)$ Individual effect of reprogramming factors on the induction of DNA damage and the expression of p53 and $\mathrm{p} 21^{\mathrm{CIP} 1}$ as measured by immunofluorescence. The expression of c-Myc and Klf4 induces p53 up-regulation. The expression of c-Myc, Klf4, or Sox2 induces the up-regulation of $\mathrm{p} 21^{\mathrm{CIP} 1}$. Similar results were obtained in two independent experiments. (O) Oct4; (S) Sox2; (K) Klf4; (M) c-Myc. (B) Expression of miRNAs of the miR-302 cluster analyzed by TaqMan in IMR90 cells infected with vector or four factors (Oct4, Sox2, Klf4, and c-Myc), three iPS cells (iPS B6, B7, and B8), and H1 hES cells (hES). (C) Expression of the miR-302 cluster (302) alleviates OSKM-induced senescence. (Left) IMR90 cells were infected with the indicated vectors and, $10 \mathrm{~d}$ post-infection, BrdU incorporation was measured after a 16-h pulse was given. (Right) Crystal violet-stained plates are shown. (Ctrl.) Control vector. $(D, E)$ miR-302a-d prevents the up-regulation of p21 CIP1 $(D)$ and p130 $(E)$ upon expression of reprogramming factors as analyzed by immunofluorescence.

(Fig. 4A). These results suggest that $\mathrm{p} 21^{\mathrm{CIP} 1}$ is activated by redundant signals during reprogramming.

Several microRNAs (miRNAs) have been identified whose expression is linked to pluripotency, such as the miR-290 cluster in mouse cells, the miR-371-373 cluster in human cells, or the miR-302 cluster conserved in both (Houbaviy et al. 2003; Suh et al. 2004). These miRNAs are expressed in ES cells and induced during the reprogramming of zygotes to the eight-cell embryo stage (Tang et al. 2007). Some of these miRNAs are necessary for the normal proliferation of ES cells, and function by targeting negative cell cycle regulators such as $\mathrm{p} 21^{\mathrm{CIP} 1}$, the $\mathrm{Rb}$ homolog p130, or LATS2 (Wang et al. 2008). In this regard, we noted that not only $\mathrm{p} 21^{\mathrm{CIP1}}$ but also the expression of p130 increased during RIS (Supplemental Fig. S11A).

The expression of the four reprogramming factors in IMR90 cells did not reset the level of these miRNAs to the ones observed in hES cells (Fig. 4B; Supplemental Fig. S12), even if the miR-302 cluster is regulated by Oct4 and Sox2 (Barroso-del Jesus et al. 2009). In contrast, iPS cells expressed these miRNAs at levels comparable with those of hES cells (Fig. 4B; Supplemental Fig. 12). Uncoupling between the expression of the four reprogramming factors and pluripotency-associated miRNAs may account for elevated p $21^{\mathrm{CIP} 1}$ expression during RIS. In accordance with our hypothesis, ectopic expression of the miR-302 cluster alleviated the growth arrest (Fig. 4C) and prevented the up-regulation of $\mathrm{p} 21^{\mathrm{CIP} 1}$ and p130 observed during RIS (Fig. 4D,E).

A corollary of our observations is that inhibition of senescence could improve reprogramming efficiency. To support this contention, we knocked down the expression of $\mathrm{p} 16^{\mathrm{INK} 4 \mathrm{a}}, \mathrm{p} 21^{\mathrm{CIP} 1}$, or p53 in BJ human fibroblasts with shRNAs (Fig. 5A). Next, we infected these cells with viruses expressing Oct-4, Sox2, Klf-4, and c-Myc and cultured them in the appropriate conditions to promote the appearance of human iPS (hiPS) cell colonies. After $21 \mathrm{~d}$, colonies were analyzed for expression of NANOG and TRA-1-60 by immunofluorescence (Supplemental Fig. 13A). Double-positive colonies were counted as fully reprogrammed iPS cell colonies, whereas morphologically distinct colonies negative for both markers were scored as partially reprogrammed (Fig. 5B; Supplemental Fig. S13A-C). Similar experiments were performed with IMR90 cells using morphological criteria to determine hiPS cell colonies and partially reprogrammed colonies. Knockdown of senescence effectors resulted in an increased number of fully and partially reprogrammed iPS cell colonies in both BJ and IMR-90 cells (Fig. 5B; Supplemental Fig. S13). Experiments carried out in MEFs in which the expression of $\mathrm{p} 16^{\text {Ink4a }} / \mathrm{p} 19^{\text {Arf }}, \mathrm{p} 21^{\text {Cip1 }}$, or $\mathrm{p} 53$ was knocked down by shRNAs, or in strains knocked out for p53 or p21, also revealed increased reprogramming efficiency upon depletion of senescence effectors (Supplemental Fig. S14).

hiPS cell colonies generated from IMR90 or BJ fibroblasts with reduced levels of senescent factors were individually picked and expanded. All the hiPS cell lines $(n=8)$ analyzed to date expressed pluripotency markers including Oct4, Sox2, Nanog, and Tra-1-60 (Fig. 5C,D; Supplemental Fig. S15), and the transgenes used for reprogramming were silenced in iPS (Fig. 5D). The hiPS lines analyzed to date $(n=8)$ were capable of differentiating in vitro into extraembryonic tissues and into derivatives of the three germ layers-ectoderm, mesoderm, and endoderm (Fig. 5E). Taken together, these data demonstrate that inhibition of senescence improves the efficiency of reprogramming of somatic cells, and the resulting iPS cells display characteristics of pluripotent stem cells.

Different reports have characterized cells that failed to fully reprogram (Pre-iPS) and suggest that they are trapped in a late step of reprogramming (Mikkelsen et al. 2008). Inhibition of DNA methylation, knockdown of lineage-specific genes, or treatment with two inhibitors (Silva et al. 2008) can either convert some of these Pre-iPS to iPS or increase the proportion of fully reprogrammed iPS versus Pre-iPS. By inhibiting or alleviating senescence, we presumably increase the cells that surpass the early barrier imposed by RIS, resulting in higher numbers 

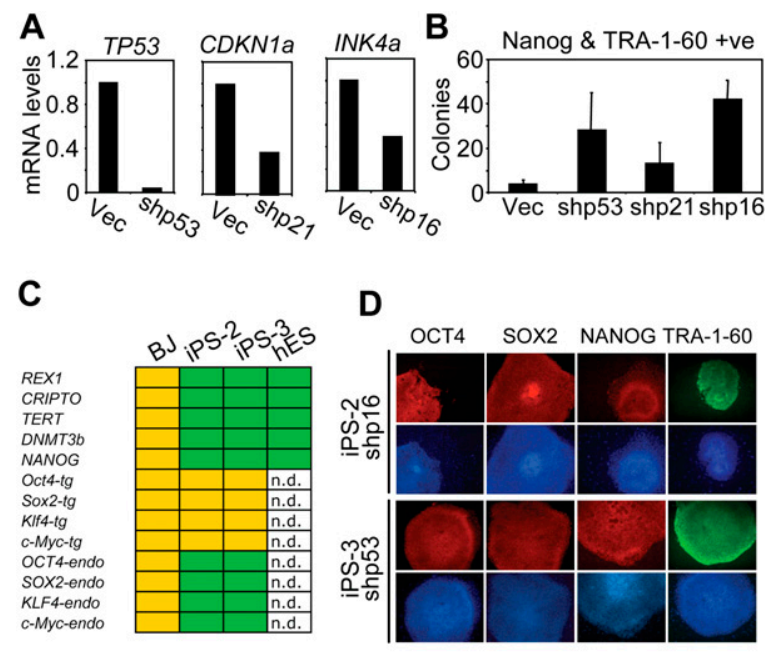

E

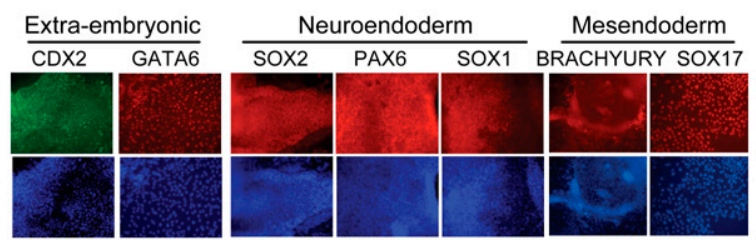

Figure 5. Inhibition of senescence improves reprogramming efficiency. (A) BJ human fibroblasts were infected with shRNAs targeting senescence effectors. Quantitative RT-PCR showing the levels of the transcripts for $\mathrm{p} 53, C D K N 1 A$ (encoding for $\mathrm{p} 21^{\mathrm{CIP} 1}$ ), and INK $4 a$ (encoding for $\left.\mathrm{p} 16^{\mathrm{INK} 4 \mathrm{a}}\right)$. (B) The BJ fibroblasts described above were transduced with lentiviruses expressing Oct-4, Sox2, Klf4, and c-Myc and grown in culture conditions compatible with pluripotent stem cell growth. Colonies were analyzed for NANOG and TRA-160 . The number of positive colonies is shown as the mean $\pm \mathrm{SD}$ of three representative experiments. $(C)$ Table summarizing the results of quantitative RT-PCR analysis of hES cell markers in iPS cells. (Green) Positive; (yellow) negative; (n.d.) nondetermined. The graphs of selected quantitative RT-PCR of this experiment are shown in Supplemental Figure 15. $(D)$ hiPS cell lines generated upon knockdown of p53 or p16 express pluripotency markers. $(E)$ hIPS cells generated upon knockdown of p53 or p16 can differentiate into extraembryonic tissues and into derivatives of the three germ layers.

of both Pre-iPS and fully reprogrammed iPS (Supplemental Fig. S16). A combination of both strategies could be used to synergistically enhance reprogramming efficiency.

In this study, we provide preliminary evidence of the pathways relevant for RIS. The up-regulation of $\mathrm{p} 16^{\mathrm{INK} 4 \mathrm{a}}$ is controlled by the epigenetic remodeling of the INK4a/ $A R F$ locus mediated, at least in part, by JMJD3. The p53/ $\mathrm{p} 21^{\mathrm{CIP} 1}$ pathway is engaged at different levels in response to the expression of reprogramming factors. Similar to what is observed during replicative-induced senescence and OIS, DNA damage is associated with the process. In addition, data extrapolated from the individual expression of reprogramming factors shows that the activation of $\mathrm{p} 21^{\mathrm{CIP} 1}$ seems a key endpoint in which different signals converge.

Reprogramming by introduction of defined factors started with empiric assays based in the knowledge of factors required for pluripotency (Takahashi and Yamanaka 2006). Additional information about the molecular mechanism behind reprogramming can be used to add logic to the method. The study of developmental reprogramming of zygotes to pluripotent embryos can render us valuable clues. miRNAs up-regulated during that process are also required to sustain the rapid cell proliferation and cell cycle profiles associated with ES cells (Wang et al. 2008). We showed that the expression of some of those miRNAs is also up-regulated in iPS but remains low at early times upon expression of reprogramming factors in fibroblasts. Ectopic expression of the miR-302 cluster alleviates RIS. Consistent with our hypothesis that inhibition of senescence enhances reprogramming efficiency, members of the miR-290 and miR-302 miRNA clusters have been shown to increase the efficiency of reprogramming (Judson et al. 2009). Although the generation of iPS is an artificial process, this exemplifies how we can learn from its mechanisms and relate them to other physiologically relevant processes.

In summary, factors and processes involved in reprogramming evoke cellular senescence. Although the link between senescence and cancer makes impractical the derivation of iPS cells lacking tumor suppressors such as p53 or p16 ${ }^{\mathrm{INK} 4 \mathrm{a}}$, treating cells during reprogramming with reversible compounds to alleviate or inhibit senescence transiently could be a feasible alternative. Defining the precise timing in which temporary inhibition of senescence is needed to improve reprogramming will be the next logical step. Besides alternative approaches, maybe transient transfection with siRNAs targeting senescence effectors could be sufficient to improve the efficiency of reprogramming. Multiple groups are concentrating their efforts on finding strategies to enhance reprogramming efficiency. In addition to unbiased screening approaches, the knowledge of the reprogramming process has already suggested compounds or genetic elements that relax epigenetic regulation or that improve the cloning efficiency of ES cells as potential candidates to enhance reprogramming. We believe that senescence will be a key process to target in the pursuit of finding more efficient strategies for deriving iPS cells.

\section{Materials and methods}

\section{Cell culture and retroviral infection}

IMR90 and BJ human primary fibroblasts were from the American Type Culture Collection. MEFs knocked out for different genes were a gift from S. Lowe (Cold Spring Harbor Laboratory). Human and mouse fibroblasts were maintained in DMEM with $10 \%$ fetal calf serum (FCS). Methods used for isolation of MEFs and retrovirus production and infection have been described (Barradas et al. 2009).

\section{Plasmids}

Vectors encoding E6, E7, E6 + E7, H-RAS ${ }^{\mathrm{V} 12 \mathrm{G}}$, or c-Myc have been described (Barradas et al. 2009). The plasmid encoding miR-302 was generated by R. Agami (NKI, Amsterdam, The Netherlands) and obtained from Geneservice. Retroviral plasmids derived from $\mathrm{pBABE}$ puro encoding reprogramming factors are listed in Supplemental Table 1.

\section{RNAi}

Vectors encoding shRNAs targeting human $\mathrm{p} 16^{\mathrm{INK} 4 \mathrm{a}}$, human $\mathrm{p} 53$, and mouse Ink4a/Arf have been described previously (Barradas et al. 2009). Sequences for shRNAs targeting human $\mathrm{p} 21^{\mathrm{CIP} 1}$ were cloned as doublestranded 21-mers in pRetroSuper, and are shown in Supplemental Table 2. 


\section{Antibodies}

The antibodies used in this study are listed in Supplemental Table 3.

\section{Immunofluorescence}

Immunofluorescence was performed using an automated high-throughput microscope (InCell Analyzer 1000, GE). Image processing and quantification was performed using InCell Investigator software (GE). A detailed description is given in the Supplemental Material.

\section{BrdU, SA $\beta$-Gal assays, and crystal violet staining}

BrdU labeling was performed for $16 \mathrm{~h}$. SA $\beta$-Gal assays and crystal violet staining were performed as described previously (Barradas et al. 2009).

\section{Quantitative RT-PCR analysis}

Quantitative RT-PCR analysis was performed using standard protocols as described in Barradas et al. (2009). The primer sets and TaqMan 6-carboxyfluorescein (FAM) Gene Expression Assays (Applied Biosystems) used are listed in Supplemental Table 2 or in Pereira et al. (2008).

\section{ChIP}

Protocols, primers, and antibodies for ChIP have been described previously (Barradas et al. 2009).

\section{Reprogramming of human and mouse fibroblasts}

Reprogramming of human and mouse fibroblasts was carried out following standard protocols. A detailed description is given in the Supplemental Material.

\section{Differentiation of hiPS cells}

Differentiation of hiPS cells was carried out following protocols developed to differentiate hES cells. A detailed description is provided in the Supplemental Materials.

\section{Acknowledgments}

We are grateful to R. Jaenisch, S. Yamanaka, and S. Lowe for supplying reagents. We also thank V. Episkopou, M. Merkenschlager, A. Fisher, and S. Raguz for critical reading. We thank K. Kaji, T. Rodríguez, M. Li, C. Bishop, M. Barradas, E. Guccione, M. Rodriguez-Niedefur, P. Hajkova, P. Svoboda, G. Peters, and D. Beach for help, encouragement, and suggestions. Investigation in L.V.'s laboratory is funded by the MRC (to L.V.), Wellcome Trust (to S.T.R.), and by NIHR Cambridge Biomedical Research Centre (to I.G.). A.B. is funded by FCT. Investigation in J.G.'s laboratory is funded by the MRC, grants from CRUK and the AICR, and by the EMBO Young Investigator Programme.

\section{References}

Barradas M, Anderton E, Acosta JC, Li S, Banito A, Rodriguez-Niedenfuhr M, Maertens G, Banck M, Zhou MM, Walsh MJ, et al. 2009. Histone demethylase JMJD3 contributes to epigenetic control of INK4a/ARF by oncogenic RAS. Genes \& Dev 23: 1177-1182.

Barroso-del Jesus A, Lucena-Aguilar G, Menendez P. 2009. The miR-302367 cluster as a potential stemness regulator in ESCs. Cell Cycle 8: 394-398.

Brambrink T, Foreman R, Welstead GG, Lengner CJ, Wernig M, Suh H, Jaenisch R. 2008. Sequential expression of pluripotency markers during direct reprogramming of mouse somatic cells. Cell Stem Cell 2: 151-159.

Carey BW, Markoulaki S, Hanna J, Saha K, Gao Q, Mitalipova M, Jaenisch R. 2009. Reprogramming of murine and human somatic cells using a single polycistronic vector. Proc Natl Acad Sci 106: 157-162.

Collado M, Blasco MA, Serrano M. 2007. Cellular senescence in cancer and aging. Cell 130: 223-233.
Feng B, Ng JH, Heng JC, Ng HH. 2009. Molecules that promote or enhance reprogramming of somatic cells to induced pluripotent stem cells. Cell Stem Cell 4: 301-312.

Houbaviy HB, Murray MF, Sharp PA. 2003. Embryonic stem cell-specific microRNAs. Dev Cell 5: 351-358.

Judson RL, Babiarz JE, Venere M, Blelloch R. 2009. Embryonic stem cellspecific microRNAs promote induced pluripotency. Nat Biotechnol 27: 459-461.

Kaji K, Norrby K, Paca A, Mileikovsky M, Mohseni P, Woltjen K. 2009. Virus-free induction of pluripotency and subsequent excision of reprogramming factors. Nature 458: 771-775.

Lowry WE, Richter L, Yachechko R, Pyle AD, Tchieu J, Sridharan R, Clark AT, Plath K. 2008. Generation of human induced pluripotent stem cells from dermal fibroblasts. Proc Natl Acad Sci 105: 28832888.

Mikkelsen TS, Hanna J, Zhang X, Ku M, Wernig M, Schorderet $\mathrm{P}$, Bernstein BE, Jaenisch R, Lander ES, Meissner A. 2008. Dissecting direct reprogramming through integrative genomic analysis. Nature 454: 49-55.

Okita K, Nakagawa M, Hyenjong H, Ichisaka T, Yamanaka S. 2008. Generation of mouse induced pluripotent stem cells without viral vectors. Science 322: 949-953.

Park IH, Zhao R, West JA, Yabuuchi A, Huo H, Ince TA, Lerou PH, Lensch MW, Daley GQ. 2008. Reprogramming of human somatic cells to pluripotency with defined factors. Nature 451: 141-146.

Pereira CF, Terranova R, Ryan NK, Santos J, Morris KJ, Cui W, Merkenschlager M, Fisher AG. 2008. Heterokaryon-based reprogramming of human B lymphocytes for pluripotency requires Oct4 but not Sox2. PLoS Genet 4: e1000170. doi: 10.1371/journal.pgen.1000170.

Silva J, Barrandon O, Nichols J, Kawaguchi J, Theunissen TW, Smith A. 2008. Promotion of reprogramming to ground state pluripotency by signal inhibition. PLoS Biol 6: e253. doi: 10.1371/journal.pbio. 0060253.

Sridharan R, Tchieu J, Mason MJ, Yachechko R, Kuoy E, Horvath S, Zhou Q, Plath K. 2009. Role of the murine reprogramming factors in the induction of pluripotency. Cell 136: 364-377.

Stadtfeld M, Nagaya M, Utikal J, Weir G, Hochedlinger K. 2008. Induced pluripotent stem cells generated without viral integration. Science 322: 945-949.

Suh MR, Lee Y, Kim JY, Kim SK, Moon SH, Lee JY, Cha KY, Chung HM, Yoon HS, Moon SY, et al. 2004. Human embryonic stem cells express a unique set of microRNAs. Dev Biol 270: 488-498.

Takahashi K, Yamanaka S. 2006. Induction of pluripotent stem cells from mouse embryonic and adult fibroblast cultures by defined factors. Cell 126: 663-676.

Takahashi K, Tanabe K, Ohnuki M, Narita M, Ichisaka T, Tomoda K, Yamanaka S. 2007. Induction of pluripotent stem cells from adult human fibroblasts by defined factors. Cell 131: 861-872.

Tang F, Kaneda M, O'Carroll D, Hajkova P, Barton SC, Sun YA, Lee C, Tarakhovsky A, Lao K, Surani MA. 2007. Maternal microRNAs are essential for mouse zygotic development. Genes \& Dev 21: 644-648.

Wang Y, Baskerville S, Shenoy A, Babiarz JE, Baehner L, Blelloch R. 2008. Embryonic stem cell-specific microRNAs regulate the G1-S transition and promote rapid proliferation. Nat Genet 40: 1478-1483.

Wilmut I, Schnieke AE, McWhir J, Kind AJ, Campbell KH. 1997. Viable offspring derived from fetal and adult mammalian cells. Nature 385: 810-813.

Ying QL, Nichols J, Evans EP, Smith AG. 2002. Changing potency by spontaneous fusion. Nature 416: 545-548.

Yu J, Vodyanik MA, Smuga-Otto K, Antosiewicz-Bourget J, Frane JL, Tian S, Nie J, Jonsdottir GA, Ruotti V, Stewart R, et al. 2007. Induced pluripotent stem cell lines derived from human somatic cells. Science 318: $1917-1920$.

Yu J, Hu K, Smuga-Otto K, Tian S, Stewart R, Slukvin II, Thomson JA. 2009. Human induced pluripotent stem cells free of vector and transgene sequences. Science 324: 797-801.

Zhou H, Wu S, Joo JY, Zhu S, Han DW, Lin T, Trauger S, Bien G, Yao S, Zhu Y, et al. 2009. Generation of induced pluripotent stem cells using recombinant proteins. Cell Stem Cell 4: 381-384. 


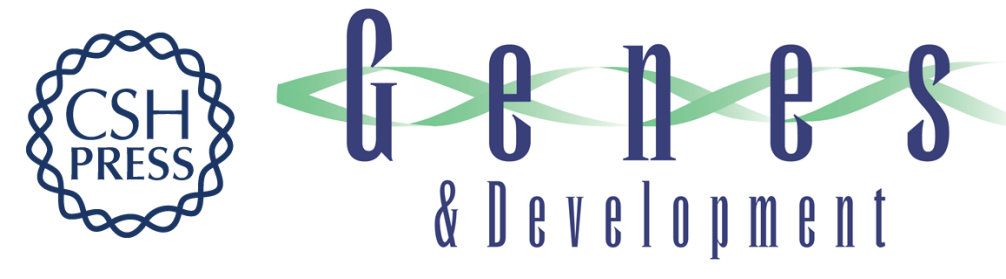

\section{Senescence impairs successful reprogramming to pluripotent stem cells}

Ana Banito, Sheikh T. Rashid, Juan Carlos Acosta, et al.

Genes Dev. 2009, 23: originally published online August 20, 2009

Access the most recent version at doi:10.1101/gad.1811609

\section{Supplemental http://genesdev.cshlp.org/content/suppl/2009/08/21/gad.1811609.DC1 Material}

References This article cites 27 articles, 8 of which can be accessed free at: http://genesdev.cshlp.org/content/23/18/2134.full.html\#ref-list-1

\section{License}

Email Alerting

Receive free email alerts when new articles cite this article - sign up in the box at the top Service

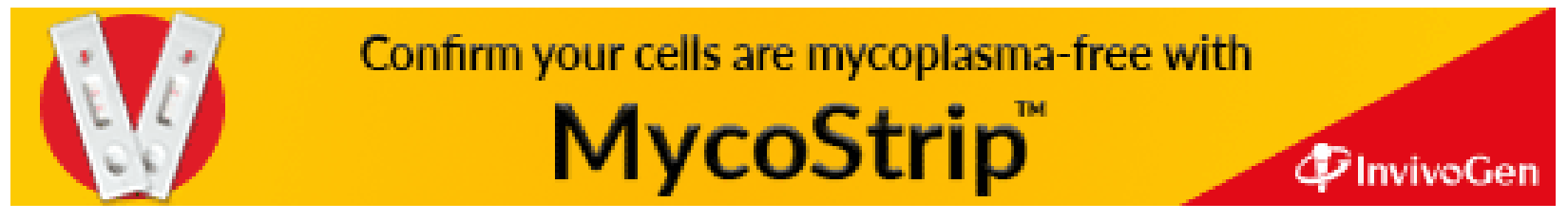

